\title{
PIN generation using EEG: a stability study
}

\author{
Ramaswamy Palaniappan* \\ School of Computer Science and Electronic Engineering, \\ University of Essex, \\ Colchester, UK \\ Email: rpalan@essex.ac.uk \\ *Corresponding author
}

\section{Kenneth Revett}

Faculty of Informatics and Computer Science, British University in Egypt, Cairo, Egypt Email: ken.revett@bue.edu.eg

\begin{abstract}
In a previous study, it has been shown that brain activity, i.e. electroencephalogram (EEG) signals, can be used to generate personal identification number (PIN). The method was based on brain-computer interface (BCI) technology using a P300-based BCI approach and showed that a single-channel EEG was sufficient to generate PIN without any error for three subjects. The advantage of this method is obviously its better fraud resistance compared to conventional methods of PIN generation such as entering the numbers using a keypad. Here, we investigate the stability of these EEG signals when used with a neural network classifier, i.e. to investigate the changes in the performance of the method over time. Our results, based on recording conducted over a period of three months, indicate that a single channel is no longer sufficient and a multiple electrode configuration is necessary to maintain acceptable performances. Alternatively, a recording session to retrain the neural network classifier can be conducted on shorter intervals, though practically this might not be viable.
\end{abstract}

Keywords: biometrics; $\mathrm{BCI}$; brain-computer interface; EEG; electroencephalogram; personal identification number; neural networks

Reference to this paper should be made as follows: Palaniappan, R. and Revett, K. (XXXX) 'PIN generation using EEG: a stability study', Int. J. Cognitive Biometrics, Vol. X, No. Y, pp.xx-xx.

Biographical notes: Ramaswamy Palaniappan's current research interests include biological signal processing, brain-computer interfaces, biometrics, artificial neural networks, genetic algorithms, and image processing. To date, he has written a book and published over 100 papers in peer-reviewed journals, book chapters, and conference proceedings. He is a senior member of the Institute of Electrical and Electronics Engineers and IEEE Engineering in Medicine and Biology Society, member in Institution of Engineering and Technology, and Biomedical Engineering Society. He also serves as editorial board member for several international journals. His pioneering work on using brain signals as biometrics has received international recognition.

Kenneth Revett is currently actively engaged in research focused in the development of the field of cognitive biometrics, which relies on the deployment of biosignals (EEG, ECG, EDR) for person authentication. He published the 


\section{R. Palaniappan and K. Revett}

first textbook dedicated to Behavioral Biometrics (Wiley \& Sons) and is currently finishing up texts in the areas of cognitive robotics, bioinformatics for computer scientists, and cognitive biometrics. He is the editor of two Inderscience journals: Int. J. of Cognitive Biometrics and the Int. J. of Cognitive Performance Support. He has authored over 130 papers (journal and conference), and served on $30+$ international programme committees.

\section{Introduction}

The advent of Automated Teller Machine (ATM) has not only enabled users to withdraw cash 24/7 from machines situated in strategic locations but also to complete many other transactions such as checking account statements to topping up pre-paid phones. Usually, users insert a card with magnetic stripe (and more recently, with a chip) into the ATMs. To authenticate the user, a matching Personal Identification Number (PIN) of four to six digits is normally entered through a small keypad. ATMs have no doubt simplified our lives in numerous ways but they also have become prone to fraud through shoulder surfing and other ingenious schemes.

PINs are not only used in ATMs but also in many other authentication devices such as those found in security door accesses and credit card readers. So, it becomes important to investigate other possible mechanisms of generating PIN that is less prone to fraud. In this light, biometric authentication has been explored, the common being the fingerprint (Wayman et al., 2004). In recent years, there have been significant interests in utilising other biometrics such as DNA, hand geometry, palm print, face (both optical and infrared), iris, retina, signature, ear shape, odour, keystroke entry pattern, gait and voice (Jain et al., 2004). However, ideas based on electrocardiogram (ECG) (Palaniappan and Krishnan, 2004) and electroencephalogram (EEG) (Palaniappan and Mandic, 2007; Palaniappan, 2008) have just started to emerge. Methods based on the use of brain electrical signals, i.e. EEG as a biometric, are relatively recent compared to the other established biometric tools.

Palaniappan and Mandic (2007) used EEG signals obtained during the perception of black and white line pictures and obtained a maximum classification accuracy of 99.38 using spectral power from Multiple Signal Classification (MUSIC) algorithm and Elman neural network classifier. It should be noted that this is the only study related to EEG biometric that was conducted with a higher number of 102 subjects. In another study, Palaniappan (2008) investigated the use of EEG extracted during mental thoughts to authenticate a person using a two-stage authentication method that gave perfect false accept rate and false reject rate when tested with five subjects. One advantage of the method was that it did not require a visual cue, thereby increasing its resistance to fraud. Furthermore, methods that require the subject to focus on visual objects that flash at certain rates (like the one proposed here) might not be suitable for users with epilepsy or similar neurological conditions.

Paranjape et al. (2001) used autoregressive (AR) features classified by discriminant analysis to achieve classification accuracy ranging between $49 \%$ and $85 \%$. Poulos et al. (1999a) also studied AR modelling of EEG signals but using Linear Vector Quantisation (LVQ) Neural Network (NN) classifier and obtained $72-80 \%$ of classification success. In another study, Poulos et al. (1999b) obtained improved classification results of $95 \%$ using the same data set but using computational geometry (convex polygon intersections) 


\section{PIN generation using EEG}

and gave an improved average classification of 95\%. More recently, Marcel and Millán (2007) analysed EEG extracted during motor imagery and word generation as a biometric. They obtained maximum accuracy of $93.4 \%$ from a data set of nine subjects. Samraj (2008) studied P300 responses from EEG as a tool for bio-cyber machine gun that can be used as an authentication tool and also to aid cryptography and information hiding by means of password shooting. However, none of these studies investigated the stability of the obtained EEG biometric over time.

In a previous study (Palaniappan et al., 2011), PIN generation using EEG signals have been proposed using only a single channel located at position $\mathrm{Cz}$. The study concentrated on minimising the number of electrodes by studying different channel configurations and the best performance was obtained from channel $\mathrm{Cz}$. This was in line with the common literature on P300 responses that are maximal in midline (Polich, 1991). Also, recent P300-based Brain-Computer Interface (BCI) studies have used location $\mathrm{Cz}$ in their investigation (Krusienski et al., 2006; Salvaris and Sepulveda, 2009). However, the stability of EEG features was not studied and we extend this idea here by investigating the stability of this biometric over time. It is crucial to have a biometric that not only gives good accuracy but also that is repeatable over time.

The method proposed here is based on the recent developments in BCI technology (Wolpaw et al., 2002). BCI designs are being developed to assist the disabled to communicate with their external surroundings. As BCI devices circumvent the peripheral nerves and muscles to create a direct link between the brain and computers/devices, they have been explored in recent times for other purposes such as games (Nijholt, 2009), virtual reality (Cho et al., 2006) and robotics (Geng et al., 2010).

There are many non-invasive BCI paradigms based on EEG signals extracted from the human scalp. Of these, single stimulus visual evoked potential method, more commonly known as the oddball paradigm method, is popular due to its ease of use by the subject with minimal training. In this method, EEG evoked for the object that the subject focused will elicit a potential known as P300 that is generated about 300-600 ms after the stimulus onset and the detection of this P300 component can be used to generate PIN based on the objects (i.e. numbers) focused by the user on screen.

The methodology behind the approach is discussed in Section 2. The next sections detail the results and discussion. The paper is concluded in Section 5.

\section{Methodology}

EEG data were collected from three right-handed male subjects aged 24-26. Four recording sessions on separate months were conducted. Each subject was briefed on the objective of the experiment before he/she signed a voluntary consent and none of the subjects had any known neurological disorder. The experiment was approved by the University of Essex's Ethics Committee. The subjects were seated in a room with computer screen projected about $30 \mathrm{~cm}$ from their eyes. All subjects had no uncorrected visual problems.

The visual stimulus paradigm is as shown in Figure 1.

Each number flashed for $100 \mathrm{~ms}$ in a random manner with $75 \mathrm{~ms}$ inter-stimulus interval (ISI) between the flashings. The ISI and flash times were chosen from a previous study (Krusienski et al., 2006). First, the subjects were told of the target number and were asked to concentrate on this target number. The subjects were suggested to keep a mental count of the target flashes to avoid lapses of concentration. This experimental paradigm 


\section{R. Palaniappan and K. Revett}

is commonly known as oddball paradigm, where the target probability is much smaller than non-target probability. Here, target/non-target ratio is $1: 10$. When a target number concentrated by the subject flashes, a positive deflection about 300-600 ms occurs after stimulus onset. This deflection will not occur for non-target cases (though, in practice, it could occur with smaller amplitude).

Figure 1 Visual stimulus paradigm

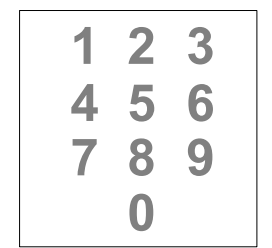

In each of the four sessions, a total of five trials were conducted where a trial consisted of ten random flashes of each number. A short break of $2.5 \mathrm{~s}$ was given between each trial. Thirty-two electrodes (locations as shown in Figure 2) were used to collect EEG with Biosemi's Active Two system. The active electrodes used in the system circumvented the requirements of cleaning the scalp prior to inserting the electrodes. However, conductive gel was still required to minimise the impedance level to below $5 \mathrm{k} \Omega$. The whole set-up required less than 15 minutes to be completed. The sampling rate used was $256 \mathrm{~Hz}$. For further processing, one second EEG data after stimulus onset of each flash were extracted.

Figure 2 Electrode configurations used in this study (a) single electrode, (b) 4 electrodes, (c) 8 electrodes, (d) 16 electrodes, (e) 32 electrodes

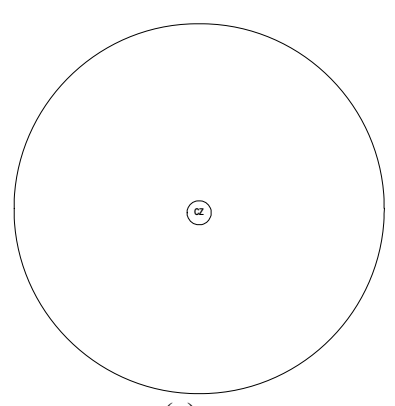

(a)

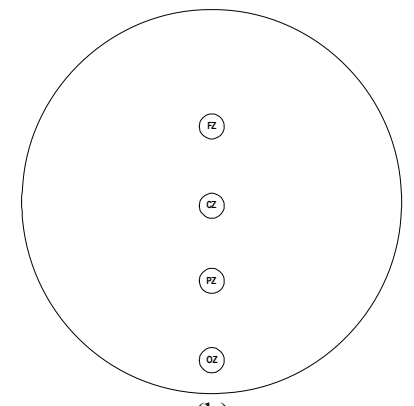

(b)

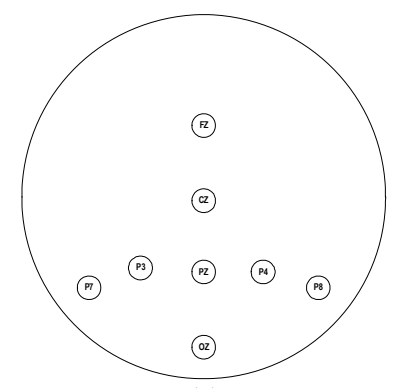

(c)

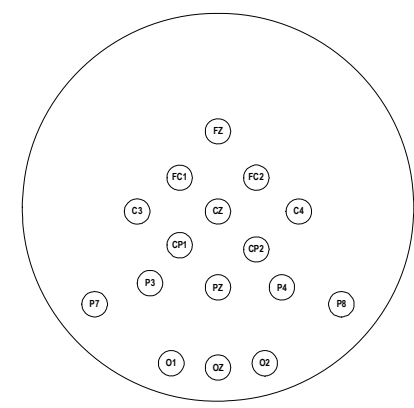

(d)

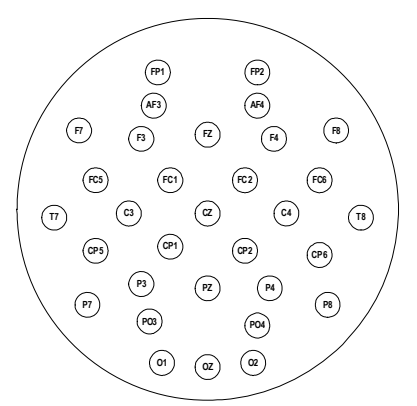

(e) 


\section{PIN generation using EEG}

A sixth-order Butterworth Infinite Impulse Response (IIR) filter was used to bandpass filter the data from 1 to $8 \mathrm{~Hz}$ as the $\mathrm{P} 300$ component is within this spectral range (Gupta and Palaniappan, 2007; Palaniappan et al., 2010). To reduce computation complexity, the data were downsampled by 8 to give 32 samples for each channel. Next, to reduce outlier data, windsorising as suggested by Hoffmann et al. (2007) was applied. Percentile values of 10 and 90 were used from each channel to cap the minimum and maximum values, respectively in the channel. Data from each channel were concatenated to give the feature vector. This processed data were given to a single hidden layer feed-forward neural network (NN) classifier. The NN was trained by the adaptive backpropagation algorithm with momentum.

Following the approach investigated by Palaniappan et al. (2011), the classifier had only two outputs, one each for target and non-target. This approach differed from the conventional approach of using ten outputs, i.e. each output for one class. Data from session one ('training session') were used to train the neural network while the data from the remaining three sessions were used separately to test the performance of the classifier. To avoid overtraining the neural network with more non-target instances as compared to target instances, all 50 target instances (from ten class of numbers and five trials) were used with 50 randomly chosen non-target instances rather than the available 450 non-target instances. NN simulations were conducted for each subject with different channel configuration as shown in Figure 2.

NN training was conducted until Mean Square Error (MSE) fell below 0.0001 or a maximum epoch size of 1000 was reached. The hidden layer size was fixed to be similar to the number of inputs. For example, when eight channels were used, the size was 256. The two outputs of the classifier were added incrementally, i.e. the outputs were aggregated after each trial. As the neural network could predict more than a single target for each trial, the maximal output after considering all the ten flashes in a trial was taken as the predicted target. The classification step was repeated ten times (to reduce effects of different neural network weight connections). Averaged performances from these ten runs were computed.

\section{Results}

Figure 3 shows the accuracy for Subject 1 after aggregated classification for first, second and third test sessions. As mentioned earlier, each test session was recorded in intervals of one month. For example, the third session was recorded after three months of recording the training session. Similarly, Figures 4 and 5 shows the results for Subjects 2 and 3.

All the subjects obtained $100 \%$ accuracy with a few different channel configurations in their first test session (i.e. after one month of recording the training data). However, the performance degraded over time for all the subjects and best performances of $90 \%$, $97 \%$ and $78 \%$ were obtained in the third test session for Subjects 1, 2 and 3, respectively. This shows that training data will need to be recorded on frequent intervals in order to maintain good performance. 


\section{R. Palaniappan and K. Revett}

Figure 3 Accuracy from Subject 1 (a) first test session, (b) second test session, (c) third test session (see online version for colour)

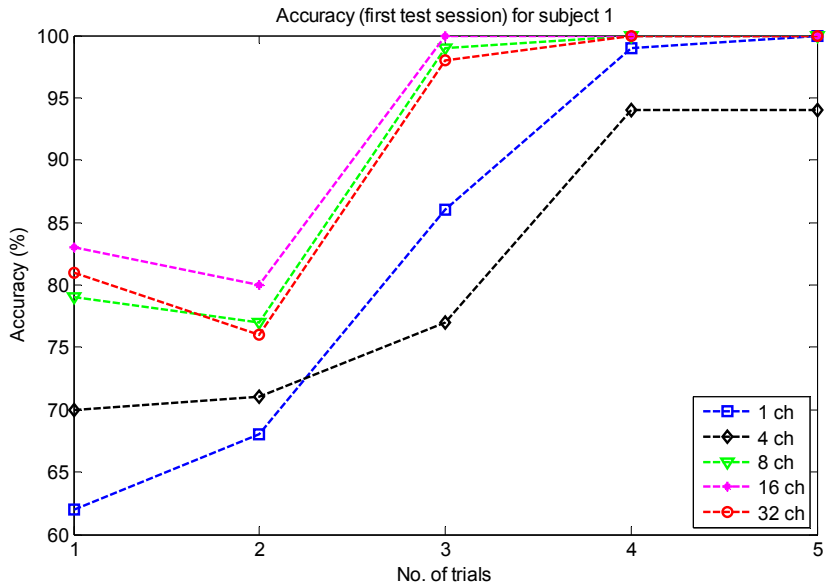

(a)

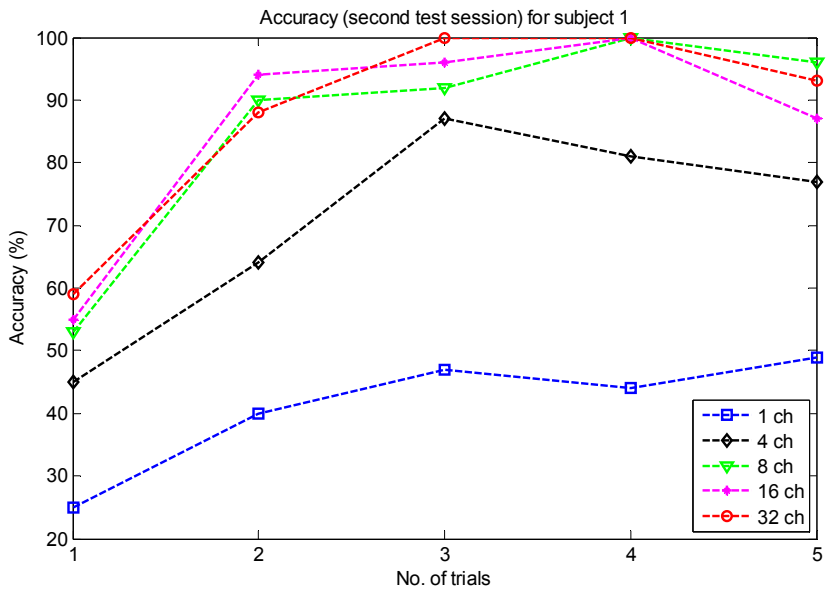

(b)

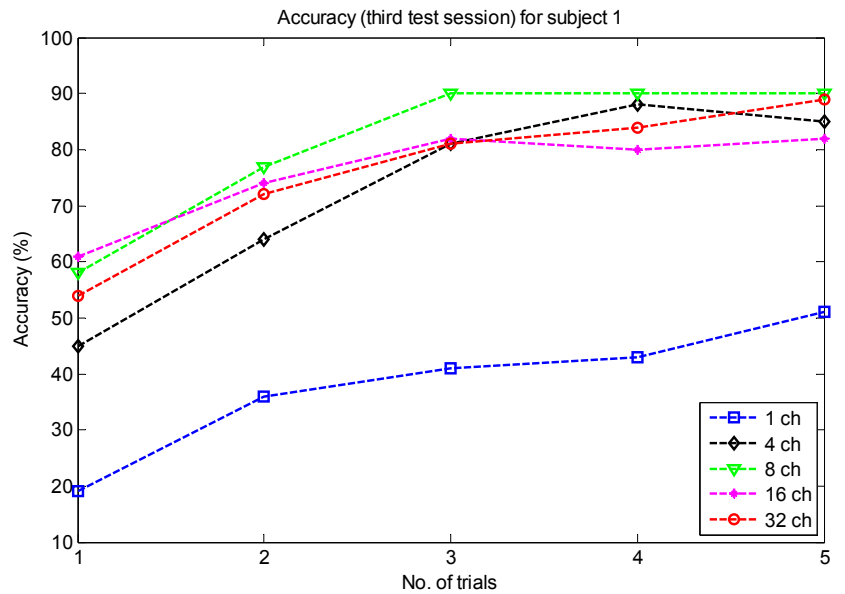

(c) 


\section{PIN generation using EEG}

Figure 4 Accuracy from Subject 2 (a) first test session, (b) second test session, (c) third test session (see online version for colour)

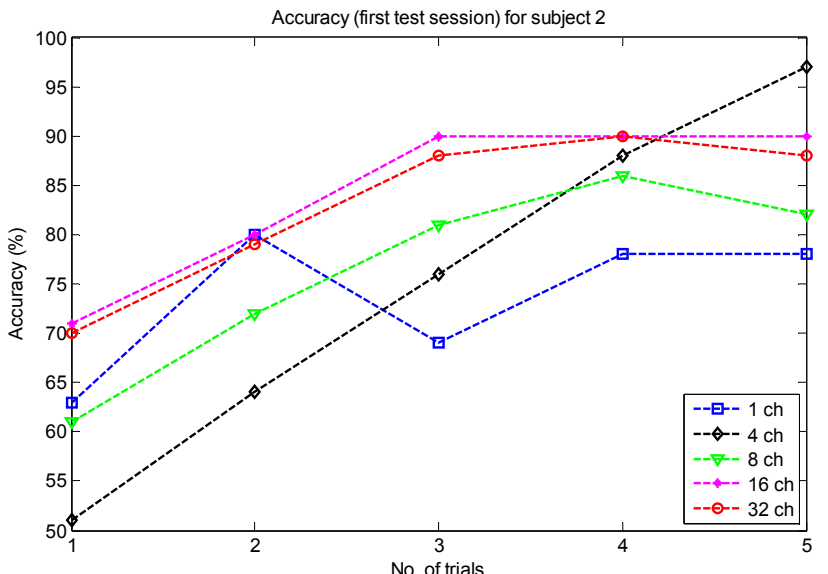

(a)

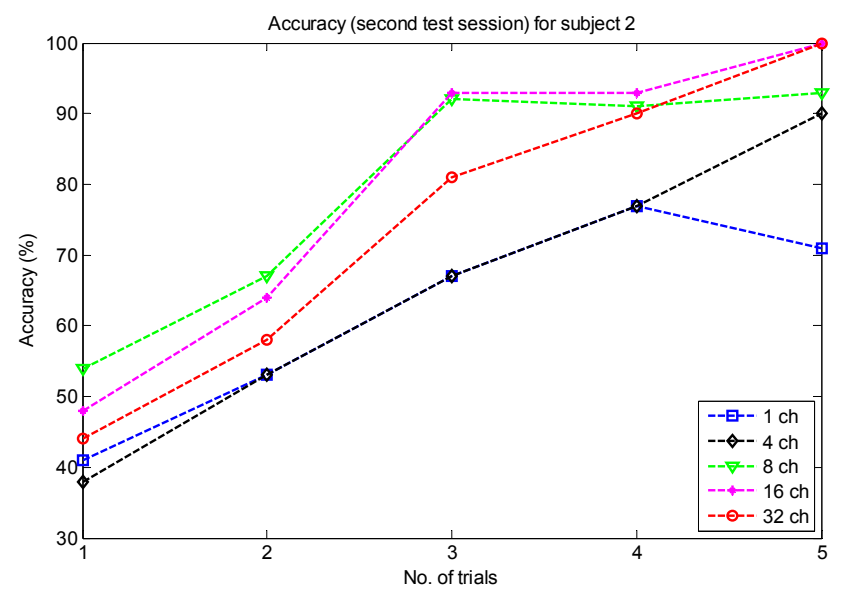

(b)

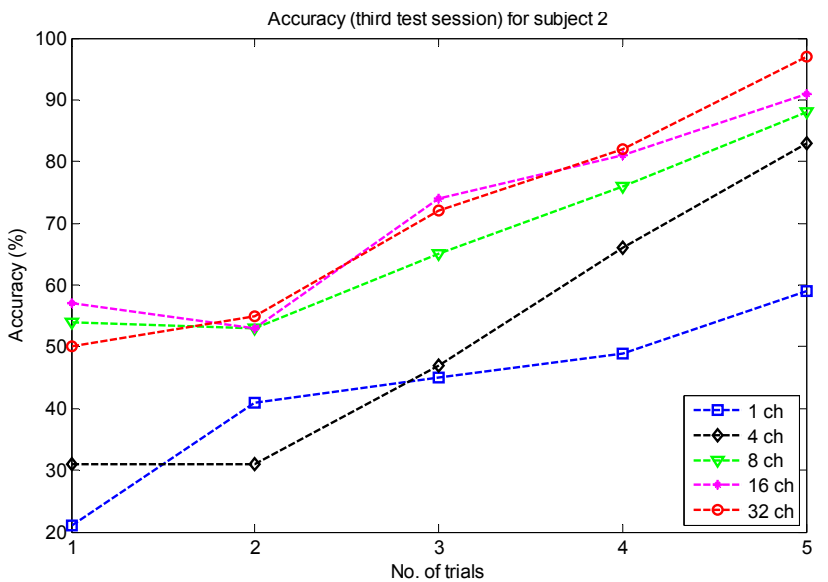

(c) 


\section{R. Palaniappan and K. Revett}

Figure 5 Accuracy from Subject 3 (a) first test session, (b) second test session, (c) third test session (see online version for colour)

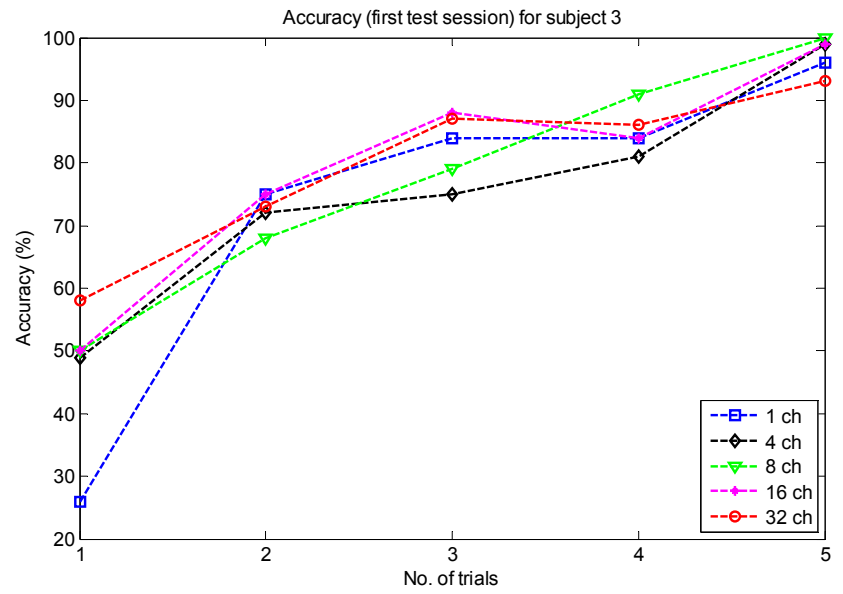

(a)

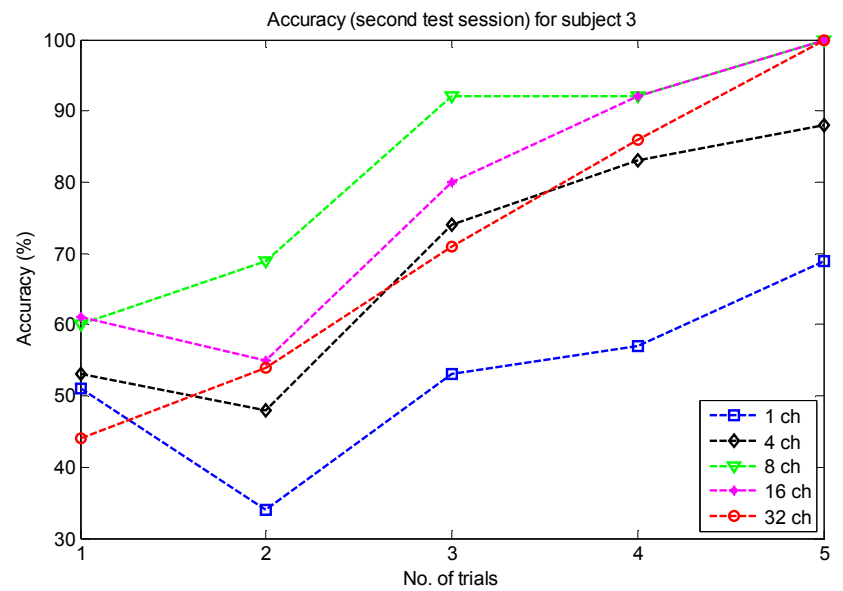

(b)

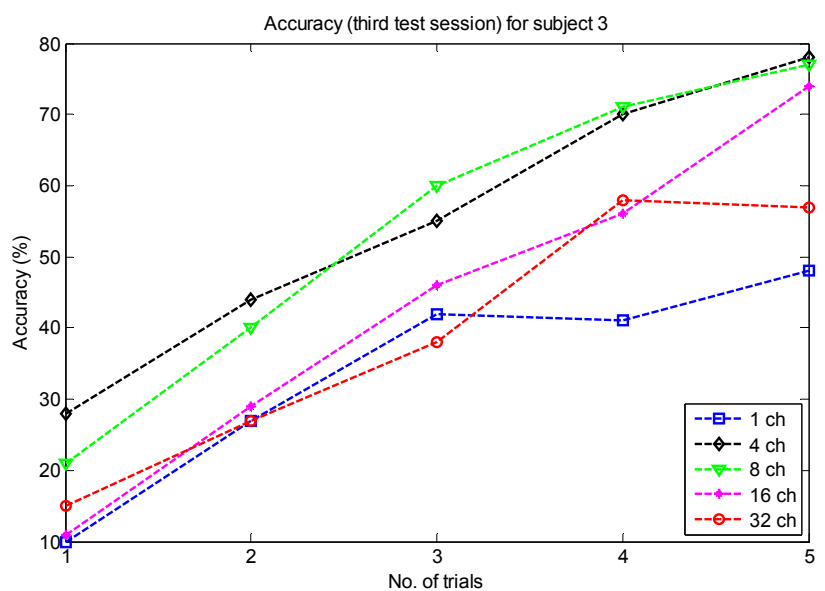

(c) 


\section{PIN generation using EEG}

Using the best accuracy obtained after five trials for third test session, the number of appropriate channels were 8, 32 and 4 for Subjects 1, 2 and 3, respectively. These results demonstrate that a single channel is not sufficient to maintain accuracy over a period of time and information from more channels helps to maintain acceptable performances. It can also be deduced that the number of optimal channels are different for different subjects. The information transfer rate (ITR) (Obermaier et al., 2001; Volosyak et al., $2010)$ in bits per minute (bpm) is a commonly used performance measure in BCI studies. It not only measures accuracy but accuracy jointly with number of targets. The bit per trial is given by

$$
B_{t}=\log _{2}(N)+P \log _{2}(P)+(1-P) \log _{2}\left(\frac{1-P}{N-1}\right)
$$

where $N$ is the number of targets and $P$ the accuracy. The bpm is given by

$$
B_{m}=\frac{60}{T} B_{t}
$$

where $T$ is the used time in seconds.

The ITR (for third test session) is given in Figure 6 using the best accuracy (for varying number of channels) for all the subjects. Subject 1 had the best ITR of $38.43 \mathrm{bpm}$ in the third session, i.e. after a period of three months from the first training session. For Subjects 2 and 3, ITR values were 33.36 and $13.15 \mathrm{bpm}$. These ITR values are comparable with those obtained by others [e.g. $20.1 \mathrm{bpm}$ as in Donchin et al. (2000) and $23.75 \mathrm{bpm}$ as in Serby et al. (2005)] but it should be noted that for biometrics, the accuracy is more important than time whereas for general BCI applications, a trade off is normally acceptable. Best ITR was obtained in the first trial for Subjects 1 and 2, while it was in fourth trial for Subject 3. As mentioned, the accuracy is more important than time for biometric studies, ITR values after fifth trial were 17.39, 20.79 and $12.78 \mathrm{bpm}$ for Subjects 1, 2 and 3, respectively. ITR values are generally lower though higher accuracies were obtained after fifth trial due to the longer time taken.

Figure 6 Best ITR values in bits/min for third test session for all the subjects (see online version for colour)

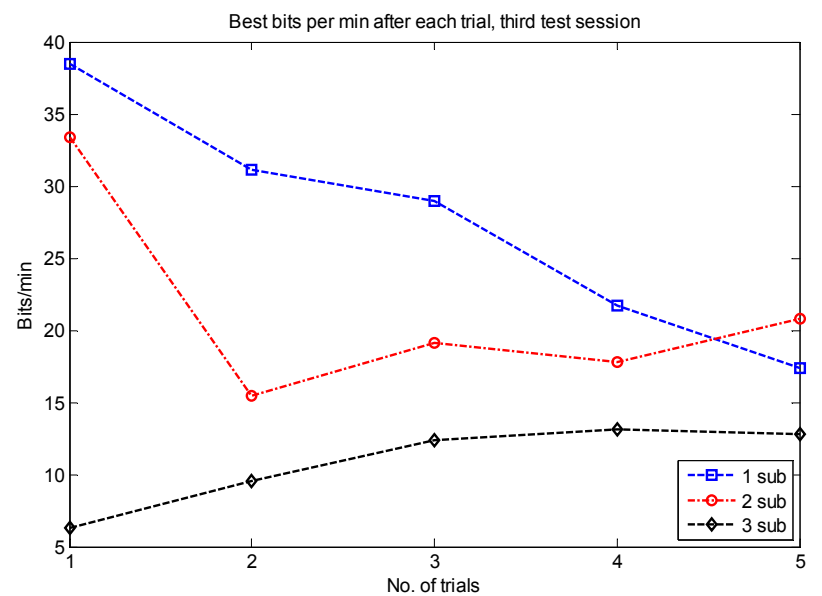




\section{R. Palaniappan and K. Revett}

\section{Discussion}

The collectability of the EEG data is an issue as it involves cumbersome wet electrodes but nevertheless should be acceptable to a large percentage of the population with the advent of dry electrodes in the near future as it is a completely non-invasive technology. Several previous studies on EEG biometric (as discussed in the Introduction section) have indicated that the EEG features are somewhat unique. However, it should be noted that the method proposed here does not require universality as it does not recognise the identity of the person but rather the object focused by the subject to generate the PIN. The results have indicated that good performance can be obtained even after three months (from training to testing session), i.e. the EEG features do indicate permanence characteristic. However, single channel $\mathrm{Cz}$ as proposed in the earlier study is no longer sufficient and information from multiple electrode configuration is necessary. As the visual cue is perceived, several areas of the brain will likely be involved to arrive at the target/non-target decision and hence the use of multiple electrodes covering more areas of the brain's responses results in stable performance over longer period of time.

\section{Conclusion}

We have investigated the stability of EEG features when classified with a neural network for purposes of generating PIN. The results have indicated that the EEG features are relatively stable, which is indicated by the good performance obtained after three months (from training to testing session). However, multiple electrode configuration is necessary to maintain the good performance over a period of time. Alternatively, a repeat of recording sessions on shorter interval would remove the requirement of multiple electrodes as zero error was obtained in the previous study with a gap of one week between training and testing. But for practical purposes, this is normally not viable and hence a multi-channel configuration as proposed here is necessary. Nevertheless, further work in this area is required to improve the performance further and to validate the method with more subjects before the proposed method can deployed for real-world applications such as accessing high-security installations.

\section{References}

Cho, H., Goo, J., Suh, D., Park, K.S. and Hahn, M. (2006) 'The virtual reality brain-computer interface system for ubiquitous home control', Lecture Notes in Computer Science, Vol. 4304, pp.992-996.

Donchin, E., Spencer, K.M. and Wijesinghe, R. (2000) 'The mental prosthesis: assessing the speed of a P300-based brain-computer interface', IEEE Transactions Rehabilitation Engineering, Vol. 8, No. 2, pp.174-179.

Geng, T., Gan, J.Q. and Hu, H. (2010) 'A self-paced online BCI for mobile robot control', International Journal of Advanced Mechatronic Systems, Vol. 2, No. 1-2, pp.28-35.

Gupta, C.N. and Palaniappan, R. (2007) 'Enhanced detection of visual evoked potentials in braincomputer interface using genetic algorithm and cyclostationary analysis', Computational Intelligence and Neuroscience, vol. 2007, pp.1-12.

Hoffmann, U., Vesin, J.M., Ebrahimi, T. and Diserens, K. (2007) 'An efficient P300-based braincomputer interface for disabled subjects', Journal of Neuroscience Methods, Vol. 167, No. 1, pp.115-125. 


\section{PIN generation using EEG}

Jain, A.K., Ross, A. and Prabhakar, S. (2004) 'An introduction to biometric recognition', IEEE Transactions on Circuits and Systems for Video Technology, Vol. 14, No. 1, pp.4-20.

Krusienski, D.J., Sellers, E. W., Cabestaing, F., Bayoudh, S., McFarland, D.J., Vaughan, T.M. and Wolpaw, J.R. (2006) 'A comparison of classification techniques for the P300 speller', Journal of Neural Engineering, Vol. 3, pp.299-305.

Marcel, S. and del R. Millán, J. (2007) 'Person authentication using brainwaves (EEG) and maximum a posteriori model adaptation', IEEE Transactions on Pattern Analysis and Machine Intelligence, Vol. 29, No. 4, pp.743-748.

Nijholt, A. (2009) 'BCI for games: a "state of the art" survey', Lecture Notes in Computer Science, Vol. 5309, pp.225-228.

Obermaier, B., Neuper, C., Guger, C. and Pfurtscheller, G. (2001) 'Information transfer rate in a five-classes brain-computer interface', IEEE Transactions on Neural Systems and Rehabilitation Engineering, Vol. 9, No. 3, pp.283-288.

Palaniappan, R. (2008) 'Two-stage biometric authentication method using thought activity brain waves', International Journal of Neural Systems, Vol. 18, No. 1, pp.59-66.

Palaniappan, R., Gosalia, J., Revett, K. and Samraj, A. (2011) 'PIN generation using single channel EEG biometric', in Abraham, A. et al. (Eds): Lecture Notes in Computer Science (International Conference on Advances in Computing and Communications), Springer-Verlag, Berlin Heidelberg. (in press)

Palaniappan R. and Krishnan, S.M. (2004) 'Identifying individuals using ECG signals', Proceedings of International Conference on Signal Processing and Communications, 11-14 December, Bangalore, India, pp. 569-572.

Palaniappan, R. and Mandic, D.P. (2007) 'Biometric from the brain electrical activity: a machine learning approach', IEEE Transactions on Pattern Analysis and Machine Intelligence, Vol. 29, No. 4, pp.738-742.

Paranjape, R.B., Mahovsky, J., Benedicenti, L. and Koles, Z. (2001) 'The electroencephalogram as a biometric', Proceedings of Canadian Conference on Electrical and Computer Engineering, Vol. 2, pp.1363-1366.

Polich, J. (1991) 'P300 in clinical applications: meaning, method and measurement', American Journal of EEG Technology, Vol. 31, pp.201-231.

Poulos, M., Rangoussi, M., Chrissikopoulos, V. and Evangelou, A. (1999a) 'Person identification based on parametric processing of the EEG', Proceedings of IEEE International Conference on Electronics, Circuits, and Systems, Vol. 1, pp.283-286.

Poulos, M., Rangoussi, M., Chrissikopoulos, V. and Evangelou, A. (1999b) 'Parametric person identification from the EEG using computational geometry', Proceedings on IEEE International Conference on Electronics, Circuits, and Systems, Vol. 2, pp.1005-1008.

Salvaris M. and Sepulveda, F. (2009) 'Visual modifications on the P300 speller BCI paradigm', Journal of Neural Engineering, Vol. 6, No. 4, p. 046011.

Samraj, A. (2008) 'Bio-cyber machine gun: a new mode of authentication access using visual evoked potentials', in Janczewski, L. and Colarik, A.M. (Eds): Cyber Warfare and Cyber Terrorism, IGI Global, Hershey, pp.338-334.

Serby, H., Yom-Tov, E. and Inbar, G.F. (2005) 'An improved P300-based brain-computer interface', IEEE Transactions on Neural Systems and Rehabilitation Engineering, Vol. 13, No. 1, pp.89-98.

Volosyak, I., Valbuena, D., Malechka, T., Peuscher, J. and Gräser, A. (2010) 'Brain-computer interface using water-based electrodes', Journal of Neural Engineering, Vol. 7, No. 6, p. 066007.

Wayman, J., Jain, A., Maltoni, D. and Maio, D. (Eds.) (2004) Biometric Systems: Technology, Design and Performance Evaluation, Springer-Verlag, London.

Wolpaw, J.R., Birbaumer, N., McFarland, D.J., Pfurtscheller, G. and Vaughan, T.M. (2002) 'Braincomputer interfaces for communication and control', Clinical Neurophysiology, Vol. 113, No. 6, pp.767-791 